\title{
Viscosity and surface-tension measurements on cyanobenzylidene octyloxyaniline using propagating capillary waves: Critical behavior
}

\author{
C. H. Sohl* \\ Argonne National Laboratory, Argonne, Illinois 60439 \\ and Department of Physics and Astronomy, Northwestern University, Evanston, Illinois 60201 \\ K. Miyano \\ Argonne National Laboratory, Argonne, Illinois 60439 \\ J. B. Ketterson \\ Argonne National Laboratory, Argonne, Illinois 60439 \\ and Department of Physics and Astronomy, Northwestern University, Evanston, Illinois 60201 \\ G. Wong \\ Department of Physics and Astronomy, Northwestern University, Evanston, Illinois 60201 \\ (Received 5 December 1979)
}

\begin{abstract}
The shear viscosities and surface tension of a liquid crystal CBOOA (cyanobenzylidene octyloxyaniline) were measured by the use of the technique of generating and detecting surface waves (capillary waves) developed by the authors. For the wave propagation perpendicular to the direction, the surface tension $\sigma$ decreased sharply upon entering into the smectic- $A$ phase. When the waves propagate parallel to the director, the attenuation due to the diverging viscosity $\eta_{3}$ near the nematic to smectic- $A$ transition became so large that measurements in the smectic phase were not possible. The viscosity $\eta_{3}$ was found to diverge as $\left(T-T_{c}\right)^{-\nu}$, where $v=1.0 \pm 0.1$ at $200 \mathrm{~Hz}$ and $v=0.83 \pm 0.15$ at $400 \mathrm{~Hz}$.
\end{abstract}

\section{INTRODUCTION}

In recent years critical phenomena have captured the attention of experimentalists and theorists alike. Experimentally such systems often exhibit spectacular pretransitional effects and pose considerable challenges to measurement techniques, while theoretically the similarities among so many diverse systems is physically intriguing. Liquid-crystalline systems exhibit critical effects associated with several different transitions ${ }^{1,2}$; a large number of studies ${ }^{3-8}$ have been made on the nematic to smectic $-A(N-A)$ phase transition in $p$-cyanobenzylidene- $p^{\prime}$-octyloxyaniline (CBOOA) because of its nearly secondorder character. Transition temperatures for $\mathrm{CBOOA}$ are given by.

solid $73.2^{\circ} \mathrm{C}$ smectic $A \stackrel{83.8}{\longleftarrow} \mathrm{C}$ nematic $107.5^{\circ} \mathrm{C}$ isotropic.

The theory for such a transition has been discussed in terms of two different models. The McMillan approach,$^{9}$ with a mean-field approximation, predicts a coherence length $\xi$ varying as $\left(T-T_{c}\right)^{-1 / 2}$ with the bend and twist elastic constants as well as the viscosities $\gamma_{1}$ and $\eta_{3}^{\prime}$ proportional to $\xi$. The de Gennes approach, ${ }^{10}$ using an analogy to the normal metal-superconductor transition, has predicted that the coherence length varies as $\left(T-T_{c}\right)^{-2 / 3}$ with the bend and twist elastic constants proportional to $\xi$ and the vis$\operatorname{cosities} \gamma_{1}$ and $\eta_{3}^{\prime}$ proportional to $\xi^{1 / 2}$. Experimentally a variety of methods have been used to study either the critical elastic constants or viscosities, and many values of the critical exponent have been reported. The diversity of reported exponenets is due in part to both differing sample purities $^{11}$ and the treatment of background contributions, which may be important if the reduced temperature, $\bar{T} \equiv\left(T-T_{c}\right) / T_{c}$, is not small. Although studies using light scattering from the thermally fluctuating $(\sim 10 \mathrm{kHz})$ free surface have been known for many years, ${ }^{12,13}$ the present study is the first to utilize low frequency $(<1 \mathrm{kHz})$ propagating surface waves and provides an interesting study of hydrodynamic theory.

In Sec. II of this paper we shall outline this theory, taken in part from a review article by Stephen and Straley ${ }^{14}$ and from a numerical and experimental study published elsewhere by us. ${ }^{15}$ In Sec. III we briefly outline the experimental techniques used. In Sec. IV we present the results of data analysis, giving our values for the viscosities $\eta_{1}^{\prime}$ and $\eta_{3}^{\prime}$ in the nematic temperature region near the $N-A$ transition, and the viscosity $\eta_{2}^{\prime}$ and surface tension $\sigma$ in both the nematic and smectic$A$ phase. An analysis of the data for the viscosity $\eta_{3}^{\prime}$ gives our values for the critical exponent. In Sec. V we discuss our results and future applicacations of our techniques. 


\section{THEORY}

The hydrodynamics of liquid crystals is developed in many texts ${ }^{16}$ and will not be reproduced here. Unlike an isotropic fluid which is characterized by a surface tension, a density, and a viscosity, a nematic liquid crystal is described by six viscosity coefficients (five of which are independent), a density $\rho$, a surface tension $\sigma$, and a unit vector $\hat{n}$ called the director, pointing in the local direction of the averaged molecular orientation. If the molecules are oriented by a magnetic field into a "nematic single crystal" then the capillary wave characteristics will depend importantly upon the relative orientation of the director $\hat{n}$ and the propagation direction $\hat{k}$ of the wave. When the magnetic field is parallel to the horizontal fluid surface, the orientations, $\hat{k} \| \hat{n}$ and $\hat{k} \perp \hat{n}$, give particularly simple predictions which we will quote. We use a Cartesian coordinate system in which the wave-propagation direction is taken to be $\hat{x}$ and the $x y$ plane $z=0$ is taken to be the equilibrium liquid free surface. The liquid occupies the volume $z<0$. We assume a plane-wave solution for the displacement from equilibrium of the liquid in the $z$ direction, 5 , of the form

$$
\zeta=\zeta_{0} e^{i(\alpha x-\omega t)} e^{m s},
$$

where the wave vector $q \equiv k+i \alpha$ is complex and the angular frequency $\omega$ is real. For waves propagating perpendicular to the molecular axis, i.e., $\overrightarrow{\mathrm{H}} \| \hat{y}$, the dispersion relation is given by ${ }^{17}$

$$
\frac{\sigma}{\rho} q^{3}-\left(\omega+\frac{i \eta_{2}^{\prime} q^{2}}{\rho}\right)-\frac{\eta_{2}^{\prime} m_{2} q^{3}}{\rho^{2}}=0
$$

where

$$
m_{2} \equiv\left(q^{2}-\frac{i \omega \rho}{\eta_{2}^{\prime}}\right)^{1 / 2},
$$

and

$$
\operatorname{Re}\left(m_{2}\right)>0
$$

is a boundary condition $\zeta=0$ at $z=-\infty$. The vis cosity $\eta_{2}^{\prime}$ is related to the Leslie viscosity ${ }^{18} \alpha_{4}$ by $\eta_{2}^{\prime}=\frac{1}{2} \alpha_{4}$. In this orientation the dispersion relation is identical to that of an isotropic fluid with parameters $\sigma, \rho$, and $\eta_{2}^{\prime}$. If the waves propagate in a direction parallel to the molecular axis, $\overrightarrow{\mathrm{H}} \| \hat{x}$, the dispersion relation is ${ }^{19}$

$$
\begin{aligned}
\sigma q^{2}\left(m_{2}^{2}-m_{1}^{2}\right)-\rho \omega^{2}\left(\frac{m_{2}^{2}+q^{2}}{m_{1}}-\frac{m_{1}^{2}+q^{2}}{m_{2}}\right) \\
-i \omega \eta_{1}^{\prime}\left(m_{1}^{2}+q^{2}\right)\left(m_{2}^{2}+q^{2}\right)\left(\frac{1}{m_{1}}-\frac{1}{m_{2}}\right)=0
\end{aligned}
$$

where

$$
\begin{aligned}
m_{1 / 2}^{2} \equiv\left(1+\frac{\eta_{3}^{\prime}}{2 \eta_{1}^{\prime}}\right) q^{2}-\frac{i \omega \rho}{2 \eta_{1}^{\prime}} \pm & {\left[q^{4}\left(\frac{\eta_{3}^{\prime 2}}{4 \eta_{1}^{\prime 2}}+\frac{\eta_{3}^{\prime}}{\eta_{1}^{\prime}}\right)\right.} \\
& \left.-\frac{i \omega \rho \eta_{3}^{\prime}}{\eta_{1}^{\prime}} q^{2}-\frac{\omega^{2} \rho^{2}}{4 \eta_{1}^{\prime 2}}\right]^{1 / 2},
\end{aligned}
$$

and $\operatorname{Re}\left(m_{1 / 2}>0\right)$, where $m_{1}$ and $m_{2}$ correspond to the + and - signs, respectively. In this orientation two fluid viscosities $\eta_{1}^{\prime}, \eta_{3}^{\prime}$ enter the dispersion relation as well as the surface tension $\sigma$ and the density $\rho$. These two viscosities are also related to those of Leslie. ${ }^{20}$

In the above discussion a number of simplifying assumptions have been made. The fluid motion has been assumed to be of low amplitude so that a linearization of the Navier-Stokes equation is legitimate. The fluid itself has been assumed to be incompressible. We have also ignored the effects of gravitation since they are significant only at low frequencies ( $<20 \mathrm{~Hz}$ in our experiment). In addition it can be shown that the effects of the Frank elastic constants and the magnetic field are negligible compared to the viscosity terms, even though the elastic constants themselves exhibit critical behavior near the $N-A$ transition.

Experimentally, by measuring the wavelength (hence wave number $k$ ) and attenuation $(\alpha)$ of surface waves of known frequency we can learn something of the material properties; e.g., for $\hat{k} \perp \overrightarrow{\mathrm{H}}, \sigma$ and $\eta_{2}^{\prime}$, and for $\hat{k} \| \overrightarrow{\mathrm{H}}, \eta_{1}^{\prime}$ and $\eta_{3}^{\prime}$. As an example we consider the perpendicular orientation in the low-viscosity limit where Eq. (2) reduces to its real and imaginary parts given by

$$
\begin{aligned}
& k^{3}=\rho \omega^{2} / \sigma, \\
& \alpha=\frac{4}{3}\left(\eta_{2}^{\prime} \omega / \sigma\right) .
\end{aligned}
$$

From these relations $\sigma$ and $\eta_{2}^{\prime}$ can be found by knowing $\rho, \omega, k$, and $\alpha$. However, for arbitrary values of viscosity, relations such as the above are not usually possible. It is therefore necessary to manipulate Eq. (2) into a form suitable for numerical solution. To do this we define two dimensionless parameters $Q$ and $P$ :

$$
\begin{aligned}
& Q \equiv q /\left(\rho \omega / \eta_{2}^{\prime}\right)^{1 / 2}, \\
& P \equiv\left(\omega \eta_{2}^{\prime 3} / \rho \sigma^{2}\right)^{1 / 2} .
\end{aligned}
$$

It can then be shown that substituting Eqs. (9) and (10) into Eq. (2) gives a dispersion relation involving a seventh-order polynomial in $Q$, as

$$
\sum_{i=0}^{7} C_{i \perp}(P) Q^{i}=0,
$$

with the dimensionless coefficients $C_{i \perp}$ being functions only of the parameter $P$. Such a polynomial, given the values of the coefficients $C_{i \perp}$, can be solved for the seven complex roots using 
the Jenkins-Traub three-stage complex algorithm. ${ }^{21}$ Nonphysical solutions are rejected. ${ }^{15}$ We have solved Eq. (11) for $P$ values between $10^{-4}$ and $10^{2}$, a range which is physically relevant to most fluids. The results appear in Fig. 1, where $Q$ is plotted as $Q=Q_{r}+i Q_{i}$ with the real $\left(Q_{r}\right)$ and imaginary $\left(Q_{i}\right)$ parts plotted simultaneously. Although there is much structure in Fig. 1 , we do not go into details since they have been discussed elsewhere along with our experimental verification of the theory using an isotropic fluid. ${ }^{15}$ It should be noted, however, that above $P=0.105$ there is a two-mode structure present consisting of a solution which approaches a critically damped wave $\left(Q_{i}=Q_{r}\right)$ and a second overdamped wave $\left(Q_{i}>Q_{r}\right)$. Such a two-mode structure has been observed on isotropic fluids using Brillouin scattering ${ }^{22}$ but our observation seems to be the first verification using a propagatingwave technique.

For the present experiment it is essential for later data analysis to estimate the mode structure of CBOOA when the waves propagate perpendicular to the molecular axis. As discussed previously, for $P<0.105$ only one mode should exist. $\mathrm{Nu}-$ merically, at a frequency of $200 \mathrm{~Hz}$ this condition is equivalent to $\eta_{2}^{\prime}<0.18$ poise if one uses the reported values for $\rho$ (Ref. 23) and $\sigma$ (Refs. 12 and 24) for CBOOA. Since the previously measured value of $\eta_{2}^{\prime}$ (Ref. 12) is less than 0.18 poise, we expect to observe a single-mode structure throughout the nematic phase. If indeed a single mode is observed we can use Fig. 1 to deduce $\eta_{2}^{\prime}$ and $\sigma$ from measurements of wave number $k$ and atten-

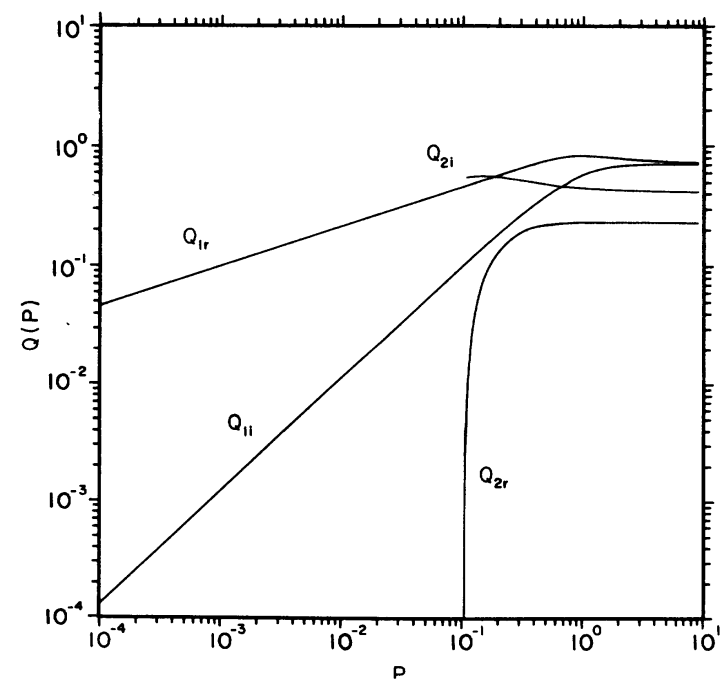

FIG. 1. Computer solution of the dispersion relation for waves propagating perpendicular to the molecular axis Eq. (11). uation $\alpha$ as follows. The ratio $k / \alpha=Q_{r} / Q_{i}$ is a monotonically decreasing function of increasing $P$. Therefore a given ratio uniquely determines $P$ and, in turn, $Q_{r}$ and $Q_{i}$. Using Eqs. (9) and (10) and known values of $\omega$ and $\rho$, one can calculate $\sigma$ and $\eta_{2}^{\prime}$ uniquely.

The analysis of surface-wave data from the $\hat{k}$ $\perp \overrightarrow{\mathrm{H}}$ orientation when the liquid crystal is in the smectic- $A$ state is identical to the analysis presented above. This is because in the perpendicular orientation a wave causes the molecules to oscillate within the smectic planes where the smectic order is not disturbed; we therefore expect that the propagation can be treated by the same
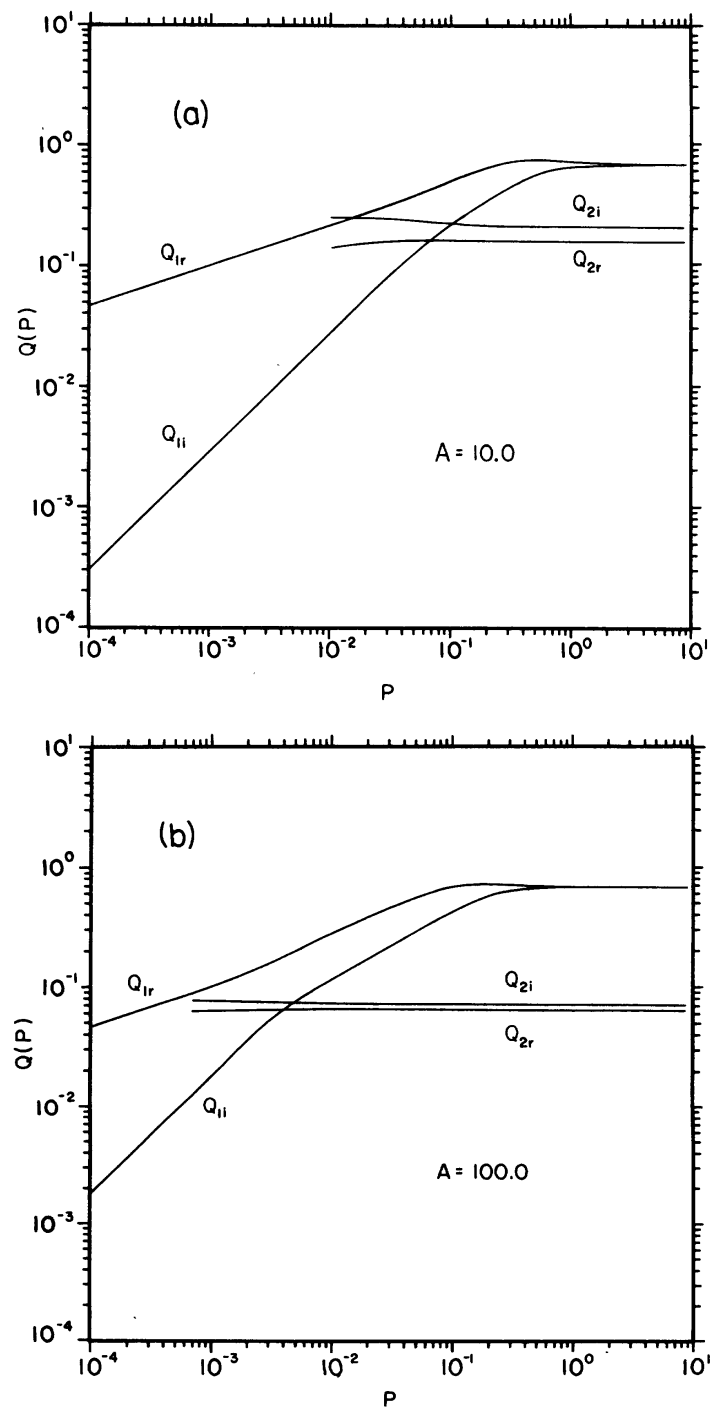

FIG. 2. Computer solution of the dispersion relation for waves propagating parallel to the molecular axis Eq. (15). (a) $A=10$; (b) $A=100$. Higher $A$ values are closer to the $N-A$ transition. 


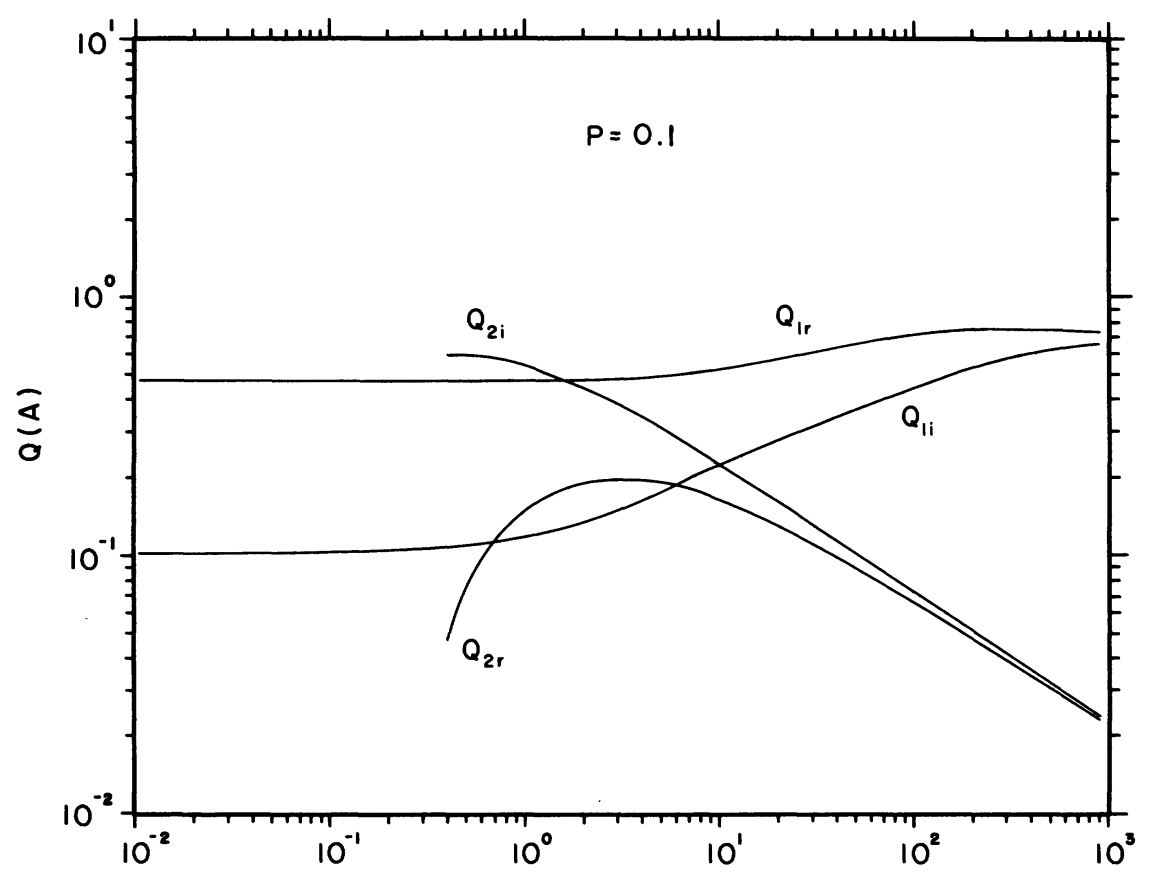

A

FIG. 3. Computer solution of the dispersion relation for waves propagating parallel to the molecular axis Eq. (15). Here the mode structure is illustrated at $P=0.1$ ( $\eta_{1}^{\prime}$ constant), and $A$ increases as $T_{c}$ is approached.

formalism as in the nematic phase.

To study the parallel orientation we must similarly deal with Eqs. (5) and (6). For arbitrary viscosity all terms contribute significantly so that a conversion to a polynomial equation is again necessary. To do this we introduce three dimensionless parameters $P, A$, and $Q$ defined as

$$
\begin{aligned}
& P \equiv\left(\omega \eta_{1}^{\prime 3} / \rho \sigma^{2}\right)^{1 / 2} \\
& A \equiv \eta_{3}^{\prime} / \eta_{1}^{\prime}
\end{aligned}
$$

and

$$
Q \equiv q /\left(\omega \rho / \eta_{1}^{\prime}\right)^{1 / 2} .
$$

After extensive manipulation Eq. (5) can then be written as

$$
\sum_{i=0}^{10} C_{i \|}(A, P)\left(Q^{2}\right)^{i}=0 ;
$$

a 10th-order polynomial in $Q^{2}$. Again unphysical solutions can be rejected and we can then plot the solutions for $Q$ as functions of $A$ and $P$. For $A=0$ the results are identical to the perpendicular calculation as expected. ${ }^{14}$ In Figs. 2(a) and 2(b) we show the results of calculations for $A=10$ and $A=100$. Note that they are qualitatively similar. It is well-known from previous studies that $\eta_{1}^{\prime}$ does not change significantly near the transition temperature (i.e., $P \simeq$ constant) while $\eta_{3}^{\prime}$ diverges. Hence a more revealing calculation which is rel- evant to the $N-A$ transition is to observe the mode structure at constant $P$ while $A$ changes. This is shown in Fig. 3 for a $P$ value of 0.1 . It can be seen that even at very low $A$ (i.e., $\eta_{3}^{\prime}$ ) values, a two-mode structure is present. We shall see in Sec. IV that this fact neccessitates a complex least-squares analysis of the surface-wave data.

\section{EXPERIMENT}

Since our system for generating and detecting capillary waves has been given elsewhere, ${ }^{25}$ only a short account will be given here. A schematic of the apparatus appears in Fig. 4. To generate the waves a sharp metal wedge is positioned very close to the fluid surface and an voltage is applied between the wedge and the fluid. The mechansim of electrocapillarity causes the fluid below the wedge to oscillate at twice the frequency of the applied voltage. The wave propagates a distance away from the generator where it causes an incident laser beam to be deflected. This deflection is detected by a position-sensing photodiode. Neither the generator nor the detector touches the fluid surface. The entire experiment is mounted on a $2300-\mathrm{kg}$ electromagnet which is supported from the floor on pneumatic cushions; hence the total mechanical noise is extremely low. The laser beam can be scanned across the surface using a mirror mounted on a translation 


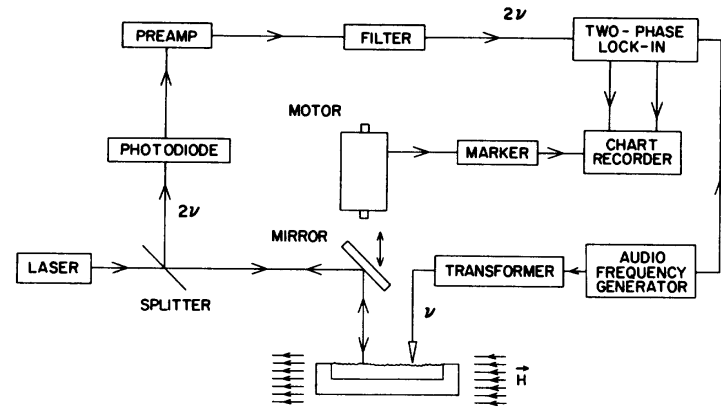

FIG. 4. Schematic of surface-wave generation and detection. The photodiode is position sensitive.

stage, and surface-wave amplitudes less than $1 \AA$ are routinely detectable.

Because liquid crystals can be oriented by electric fields,$^{16}$ the distorting effects of the intense electric fields near the tip of the wedge on the magnetically aligned sample must be discussed. Even though an exact calculation of the electric-field strength is difficult because of the geometry, it is clear that with a small gap (near $0.05 \mathrm{~mm}$ in our experiment) and with applied potentials near 50 volts, the fields near the wedge are exerting strong orienting forces on the molecules. It is expected, however, that these fields are not present in the regions of the sample where surface-wave behavior is studied; even when focussed the diameter of the laser beam (about $0.5 \mathrm{~mm}$ ) prevents study of the surface at distances less than $0.5 \mathrm{~mm}$ from the wedge, roughly ten gap widths away. At these distances from the wedge, the field decays to a small fraction of its value directly beneath the wedge. There is thus no reason to suspect that in the regions studied the liquid-crystalline order deviates in any sigsificant way from that imposed by the magnetic field. It is, in fact, seldom necessary to go so near the wedge since the apparatus is very sensitive. I In addition, the more important effects of the stationary rise ${ }^{25}$ of the surface near the wedge makes surface-wave measurements there very difficult and unreliable; hence these regions are avoided.

Although the critical behavior of the viscosity at the $N-A$ transition has been studied using Brillouin scattering from thermally excited surface waves $(\sim 10 \mathrm{kHz})$, the present experiment is the first to study such behavior using low-frequency capillary waves. Previous methods ${ }^{26}$ of generating and detecting such propagating waves using speaker coils and phonograph pickups are unworkable in the confined geometries imposed by a high-field electromagnet and by temperature regulation requirements. In addition such methods would destroy the liquid-crystalline order. In contrast our apparatus is free of these problems and is ideally suited for the present study.

Experiments on CBOOA were carried out in both the $\hat{k} \perp \overrightarrow{\mathrm{H}}$ and $\hat{k} \| \overrightarrow{\mathrm{H}}$ orientations using a $10-\mathrm{kG}$ field. We varied the temperature from the nematic to the smectic phase with about 20 minutes between points to assure thermal equilibrium. Temperature regulation was better than $0.01{ }^{\circ} \mathrm{C}$. At each temperature data were taken at both 200 and 400 $\mathrm{Hz}$. Because the waves decay quickly with distance from the generator, reflections from the side walls of the trough are unobservable. Reflections from the bottom could be non-negligible because at temperatures near $T_{c}$ where the longwavelength mode becomes important, the ratio of wavelength to depth $(\lambda / d)$ is not small. However, we do not consider depth effects to be significant since in our previous study of isotropic fluids we did not see appreciable differences between the data taken in a shallow trough $(\lambda / d \sim 0.3)$ and those taken in a deeper one $(\lambda / d \ll 1)$.

\section{ANALYSIS}

Actual data consist of the output of a two-phase lock-in; one channel gives a signal $d_{2}(x)$ in quadrature to a signal $d_{1}(x)$ in another channel. Both signals are analyzed simultaneously in order to increase the accuracy of the fitting parameters. For our experiment the data were assumed to be of the form

$$
\begin{aligned}
& d_{1}(x, n)=\sum_{i=1}^{n} a_{i} e^{-\alpha_{i} x} \cos \left(k_{i} x+\phi_{i}\right), \\
& d_{2}(x, n)=\sum_{i=1}^{n} a_{i} e^{-\alpha_{i} x} \cos \left(k_{i} x+\phi_{i}-\pi / 2\right),
\end{aligned}
$$

where $n=1$ if only one mode is present $(\overrightarrow{\mathrm{k}} \perp \overrightarrow{\mathrm{H}})$ and $n=2$ if two modes $(\hat{k} \| \overrightarrow{\mathrm{H}})$ are present. The $a_{i}$ are proportional to the amplitudes of the waves, the $\alpha_{i}, k_{i}$, and $\phi_{i}$ are the wave attenuations, wave numbers, and phases, respectively. Data analysis consists of extracting $\alpha_{i}$ and $k_{i}$ from the experimental traces and then deducing the related fluid parameters. The case $n=1$ has already been discussed in the theory section. The analysis of a trace in which $n=2$ is much more difficult and we use the following procedure. At any given temperature the fluid density is known from other experiments, and the surface tension is known from our own analysis of the perpendicular orientation because the surface tension should be isotropic in the nematic phase. With an initial guess for $\eta_{1}^{\prime}$ and $\eta_{3}^{\prime}$, and knowing the surface wave frequency, we use definitions (12) and (13) to form our initial guess for the parameters $A$ and $P$. With $A$ and $P$ known we can solve the dispersion 

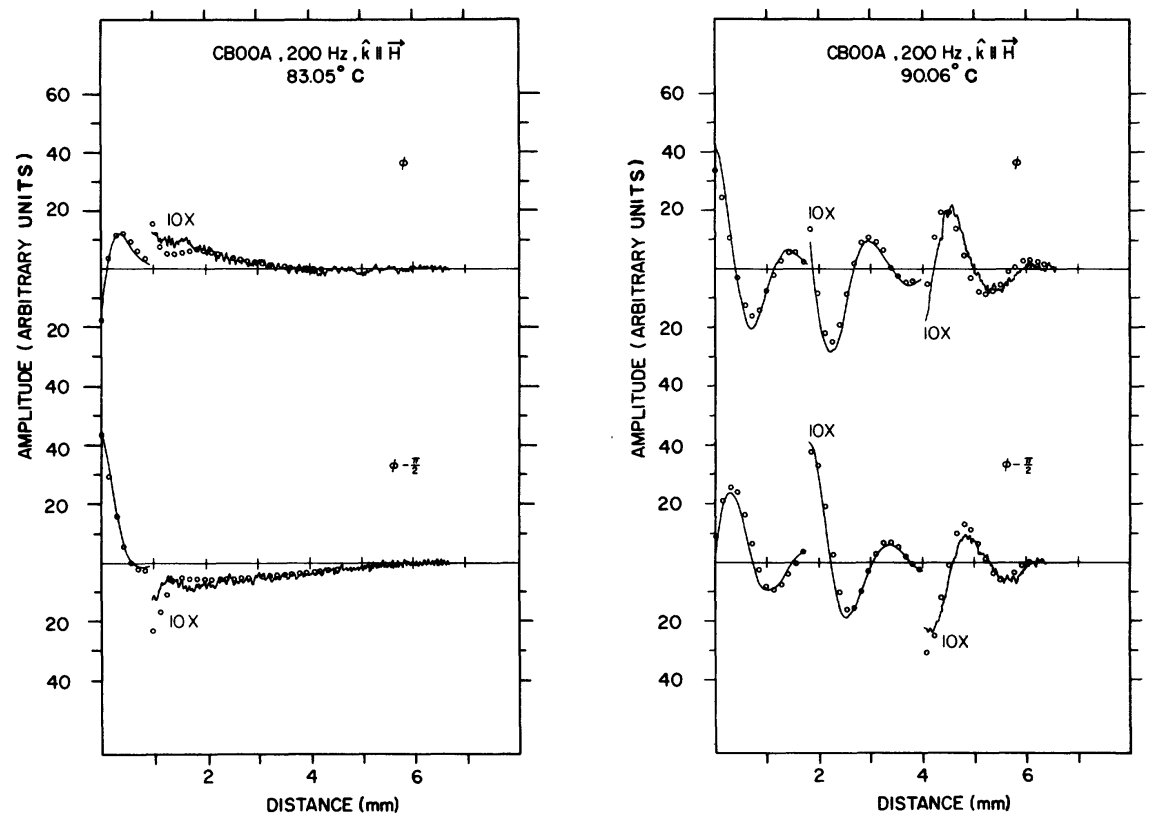

FIG. 5. Typical data from the $\vec{k} \| \overrightarrow{\mathrm{H}}$ orientation. The circles are the least-squares fits. Both channels are shown.

relation for $Q_{1}$ and $Q_{2}$ and from definition (14) we then have $q_{1}$ and $q_{2}$. We have thus found a guess as to what waves are to be used to fit the two data channels. We can then vary the wave amplitudes $a_{i}$ and phases $\phi_{i}$ until a rough fit to both channels is obtained. This fit forms an initial guess in a nonlinear least-squares analysis of the traces in which the six parameters, which we shall write as $\overrightarrow{\mathrm{P}}=\left\{A, P, a_{1}, a_{2}, \phi_{1}, \phi_{2}\right\}$, are adjusted to achieve the best fit (to be defined below). Because $\alpha$ and $k$ are functions of $A$ and $P$ through Eq. (15), the right-hand side of Eqs. (16) and (17) can be rewritten as

$$
g(x, \overrightarrow{\mathrm{P}})
$$

and

$$
g(x, \overrightarrow{\mathrm{P}}-\{0,0,0,0, \pi / 2, \pi / 2\}) \equiv g^{\prime}(x, \overrightarrow{\mathrm{P}})
$$

for $n=2$, respectively. We then demand that the parameters $\overrightarrow{\mathbf{P}}$ be adjusted to fit both data channels simultaneously so as to minimize the following function:

$$
\begin{aligned}
f= & \sum_{i=1}^{m}\left[d_{1}\left(x_{i}\right)-g\left(x_{i}, \overrightarrow{\mathrm{P}}\right)\right]^{2} \\
& +\sum_{i=1}^{m}\left[d_{2}\left(x_{i}\right)-g^{\prime}\left(x_{i}, \overrightarrow{\mathrm{P}}\right)\right]^{2},
\end{aligned}
$$

where $m$ is the number of points digitized from the experimental traces $d_{1}$ and $d_{2}$. There are two points worth emphasizing about this procedure.
(1) We have used the surface tension found from the $\hat{k} \perp \overrightarrow{\mathrm{H}}$ analysis as a known value in the $\hat{k} \| \overrightarrow{\mathrm{H}}$ analysis. (2) The parameters $A$ and $P$ determine $\alpha_{i}$ and $k_{i}$ implicitly through the dispersion relation (15); hence the values $\alpha_{i}$ and $k_{i}$ are not independent parameters. When least-squares convergence was achieved, $\eta_{1}^{\prime}$ and $\eta_{3}^{\prime}$ were calculated using the parameters $A$ and $P$ and the definitions (12) and (13).

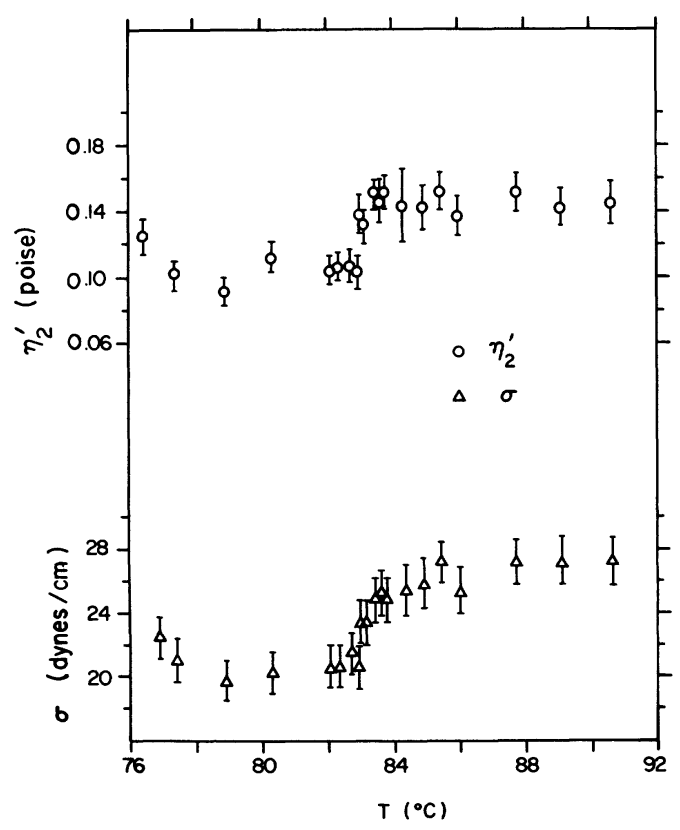

FIG. 6. Results of analysis of $\overrightarrow{\mathrm{k}} \perp \overrightarrow{\mathrm{H}}$ data at $200 \mathrm{~Hz}$. 
It is interesting to show how the nature of the waves changes in agreement with our calculations as the transition temperature is approached. Far from $T_{c}$ at $90.06{ }^{\circ} \mathrm{C}$ we show in Fig. 5 an experimental trace and the fit provided from the above procedure. The trace is composed of predominantly a short-wavelength low-attenuation mode $\left[Q_{1}\right.$ in Fig. 2(a)]. At $83.05^{\circ} \mathrm{C}$ we see a substantial contribution from the $Q_{1}$ mode close to the generator along with a dominant contribution from the $Q_{2}$ mode, a long-wavelength mode further away from the generator.' Thus using the above methods we were able to extract results for $\eta_{1}^{\prime}$, $\eta_{2}^{\prime}, \eta_{3}^{\prime}$, and $\sigma$.

\section{DISCUSSION}

Results from the perpendicular orientation ( $\sigma$ and $\eta_{2}^{\prime}$ ) are shown in Fig. 6, while the results of our least-squares analysis of the parallel orientation appear in Fig. 7. Together these two figures represent a complete set of parameters for CBOOA versus temperature. At the present time we are aware of only one other experiment (Langevin ${ }^{12}$ ) in which $\eta_{1,2,3}^{\prime}$ and $\sigma$ are simultaneously measured for $C B O O A$ in an orienting field. Our results for the temperature variation of $\eta_{2}^{\prime}$ agree with those of Langevin in the nematic temperature range although we have observed a dip in the viscosity $\eta_{2}^{\prime}$ at the transition to the smectic- $A$ phase. Langevin reports (footnote 2 of Ref. 12) that the surface tension $\sigma$ remained constant at $27 \pm 2 \mathrm{dyn} / \mathrm{cm}$ throughout the nematic phase. Again our results are in agreement in the nematic phase. In addition we have made surface-tension measurements in the smectic- $A$ phase and again have observed a change across the transition. At present we are unaware of any other measurements of the surface tension of a smectic- $A$ liquid crystal in a magnetic field. Unaligned studies were made on CBOOA using the pendant-drop method by Krishnaswamy and Shashidhar ${ }^{24}$ who reported a linear decrease in surface tension upon transition to the smectic phase; their data in the smectic state is however not in quantitative agreement with our results. They have also reported a surface tension near $27 \mathrm{dyn} / \mathrm{cm}$ throughout the nematic phase. Gannon and Faber ${ }^{27}$ have recently reported unaligned measurements of surface tension at the nematic to isotropic transition on 4-n-pentyl-4'cyanobiphenyl (5 CB) and 4-n-octyl-4'-cyanobiphenyl $(8 \mathrm{CB})$ using a Wilhelmy plate technique. They observed increases of surface tension upon going from the nematic (ordered) to isotropic (disordered) states in agreement with observations of Krishnaswamy and Shashidhar on CBOOA. We have also studied the nematic to isotropic transition on CBOOA and have seen a positive jump in surface tension upon entering the isotropic phase with a negative slope of the $\sigma$ versus $T$ curve in the isotropic phase. It is reasonable to expect that in going from the ordered state (smectic) to the less ordered state (nematic) the surface energy again increases (reflecting the increase of disorder) as we have observed in Fig. 6. In fact from the Maxwell relation for surface tension

$$
-\left(\frac{\partial \sigma}{\partial T}\right)_{A^{\prime}}=\left(\frac{\partial S}{\partial A^{\jmath}}\right)_{T}
$$
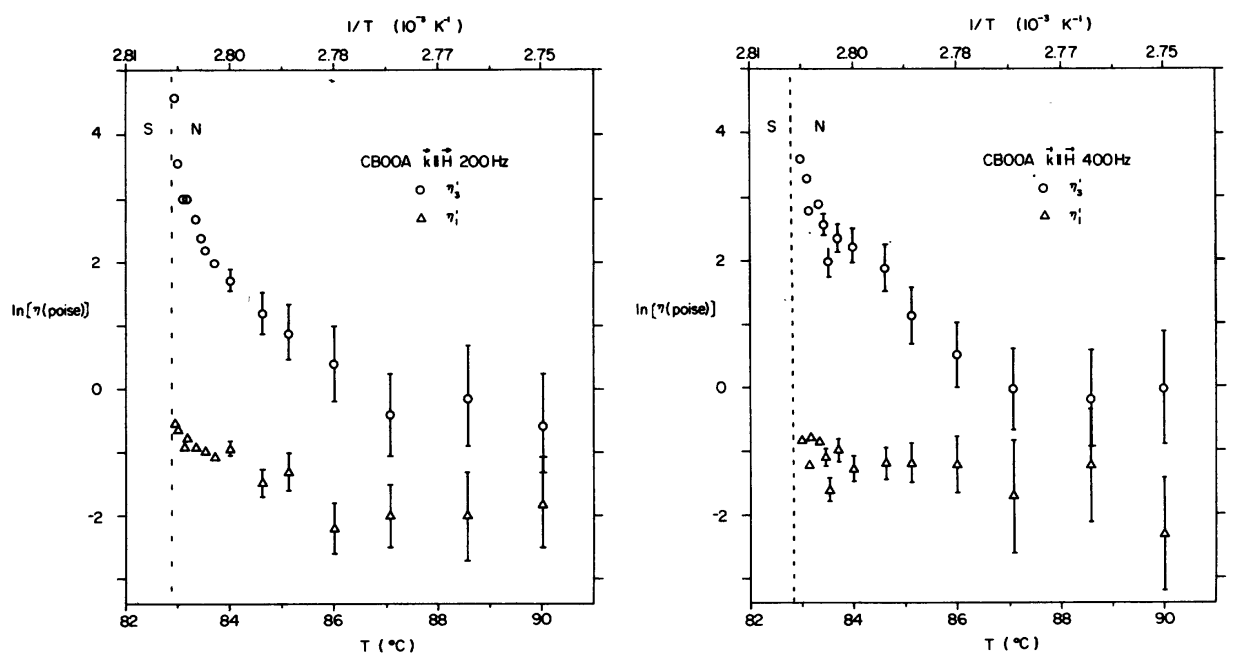

FIG. 7. Results of least-squares analysis of $\vec{k} \| \vec{H}$ data. 
where $\sigma$ is surface tension, $T$ temperature, $S$ entropy, and $A^{\prime}$ surface area, we can see that if the slope of the surface tension-temperature plot is positive the entropy decreases as the area increases. This indicates that the surface region is more ordered than the bulk. From our data we can then argue that such a situation does exist and that (as speculated by Gannon and Faber) the excess order might be due either to an increased order parameter $\left\langle P_{2}(\cos \theta)\right\rangle$ at the nematic surface, or in fact the beginnings of smectic ordering.

Our results for $\eta_{1}^{\prime}$ are also in agreement with Langevin's data for $\eta_{1}^{\prime}$. The error bars shown on the viscosities of Fig. 7 are dominantly due to the uncertainties in the surface tension which was used in the least-squares fitting. The error bars on $\sigma$ and $\eta_{2}^{\prime}$ are due to the uncertainties in the measurements of the attenuations and wavelengths.

Close to the transition temperature where $\eta_{3}^{\prime}$ $\gg \eta_{1}^{\prime}$ the error bars on the viscosities are near the actual symbol size. Because at high $\eta_{3}^{\prime}$ the mode structure is primarily determined by $\eta_{3}^{\prime}$, the fitting procedure converges more rapidly and with less uncertainty in the final values for $\eta_{1,3}^{\prime}$ than the case where $\eta_{1}^{\prime}$ and $\eta_{3}^{\prime}$ are comparable, such as at $T=90^{\circ} \mathrm{C}$. In the latter case both viscosities are equally important and it is difficult to separate the contribution due to each. The situation is analogous to that in surface-wave Brillouin-scattering experiments where the halfwidth $(\Delta \nu)$ of the power spectrum of surface fluctuations is given (in the low-viscosity limit) by

$$
\Delta \nu=\frac{\eta_{1}^{\prime}+\frac{1}{8} \eta_{3}^{\prime}}{\pi} \frac{q^{2}}{\rho}
$$

for wave vector $\vec{q} \| \vec{H}$ (Ref. 28); the halfwidth may be measured experimentally but $\eta_{1}^{\prime}$ and $\eta_{3}^{\prime}$ by themselves are undetermined.

In Fig. 7 we have shown data at two frequencies: 200 and $400 \mathrm{~Hz}$, respectively. In Fig. 8 we have analyzed the temperature dependence of the critical viscosity $\eta_{3}^{\prime}$. To do this we have used the standard procedure where $\eta_{3}^{\prime}$ is written as the sum of two contributions: a background Arrhenius contribution $\eta_{30} \sim e^{B / T}$ and a divergent contribution $\tilde{\eta}_{3} \sim\left(T-T_{c}\right) \nu$ with $\nu$ being the critical exponent. Because of the large scatter in the high-temperature data for $\eta_{3}^{\prime}$, the background contribution $e^{B / T}$ was estimated from capillary viscometer data ${ }^{29}$ where the scatter was much smaller. It was found however that the final fit to the data was not sensitive to a choice in background parameters. Upon performing least-squares analysis of both frequencies we have found that for the $200-\mathrm{Hz}$ data the critical exponent is given by $\nu=1.0 \pm 0.1$ with a transition temperature $T_{c}=82.96{ }^{\circ} \mathrm{C}$, while at $400 \mathrm{~Hz}$ we find that the critical exponent is given by $\nu=0.83 \pm 0.15$ with $T_{c}=82.90^{\circ} \mathrm{C}$. Because these values for the exponent are much larger than those reported in the literature ${ }^{30}$ we will consider possible causes of the discrepancy.

First, it is unlikely that the impurities are the cause because of the following reasons. Optically the transition temperature appears at $\mathbf{8 2 . 9}$ $\sim 83.0^{\circ} \mathrm{C}$. Some studies ${ }^{11}$ have shown that large exponents can be obtained by impurity doping of a pure sample, while other studies ${ }^{31}$ report that impurities do not affect the order of the transition. We have previously reported ${ }^{32}$ a high exponent $(\nu=1.0)$ on a different sample of CBOOA whose surface tension was about $22 \mathrm{dyn} / \mathrm{cm}$ in the nematic phase. Because such a low surface tension can be interpreted as evidence of impurities, we performed the experiment again on the
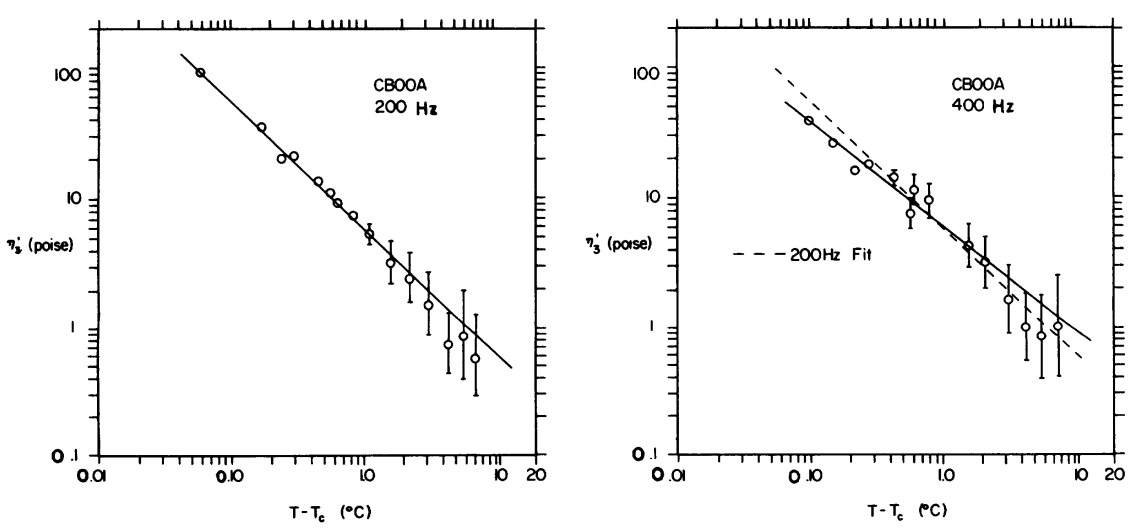

FIG. 8. Critical plots of $\eta_{3}^{\prime}(T)$ versus $T-T_{c}$. At $200 \mathrm{~Hz}$ least-squares analysis gives $T_{c}=82.96^{\circ} \mathrm{C}$ with $\nu=1.0 \pm 0.10$ while at $400 \mathrm{~Hz} T_{c}=82.9^{\circ} \mathrm{C}$ with $\nu=0.83 \pm 0.15$. 
current sample. Since our current value of the surface tension indicates the absence of surfaceactive impurities the high value of the exponent is not due to impurities of this type. Moreover the transition temperature of our sample suggests that the sample is relatively pure according to Fig. 2 of Ref. 11.

The nonparallel anchoring of molecules at the surface should not affect our measurement in the nematic phase. The aligning magnetic field is strong enough $(\sim 1 \mathrm{~T})$ so that the healing length of the director should remain much shorter $(<10 \mu \mathrm{m})$ than the wavelength of the capillary wave $(\sim 1 \mathrm{~mm})$ throughout the nematic phase. Moreover this phenomenon should affect the results of lightscattering experiments ${ }^{12}$ more because of shorter wavelengths $(\sim 0.3 \mathrm{~mm})$ and lower magnetic fields $(\sim 0.3 \mathrm{~T})$ employed there. Thus the discrepancy of the exponent to the reported value ${ }^{12}$ of 0.54 \pm 0.08 cannot be explained by this mechanism. The anchoring, however, can be a problem in the smectic $-A$ phase, where the texture of the surface is different ${ }^{33}$ from that of the bulk. In fact, the surface roughness caused by the focal conic texture was large for 40.8 (butoxybenzylidene-octylaniline) and for DEAB (diethylazoxybenzoate) that we could not obtain a well-defined reflected light from the surface. Although the surface roughness was not a problem for $\mathrm{CBOOA}$, this point should be kept in mind in interpreting Fig. 6 in the smectic region.

The present measurements are different from the work ${ }^{12}$ by Langevin in that we study the propagation characteristics of externally generated waves. We, therefore, use relatively large amplitude waves: for example, the amplitude of the waves $^{25}$ at the origin of Fig. 5 is about $100 \AA$. However, the nonlinearity in the hydrodynamic equation should be negligible since $a k \ll 1$, where $a$ is the amplitude and $k$ is the wave number.

It is interesting to note that a viscosity measured by a capillary viscometer shows ${ }^{29}$ a divergence with an exponent near unity. It is possible that an additional relaxational effect comes in at low frequencies $(<1 \mathrm{kHz})$. However, this is the frequency region where dynamical studies are scarce and further evidence is clearly needed.

In closing it is worth mentioning that the techniques developed by us are not limited to bulk liquid-crystal studies. We are currently engaged in experiments attempting to detect two-dimensional attenuation anisotropy by magnetically aligning surface films of monomolecular systems spread on water substratęs. Such experiments, if successful, could provide important studies of current theories of two-dimensional order and phase transitions.

\section{ACKNOWLEDGMENTS}

This research has been performed under the auspices of the United States Department of Energy and supported in part by the National Science Foundation under Grant No. DMR-76-21370. We thank D. D. Koelling for assistance in computationrelated problems. One of us (C.H.S.) would like to thank the Argonne Center for Educational Affairs for thesis support.
*Present address: E. I. DuPont de Nemours \& Co., Inc. Plastic Products and Resins Division, Experimental Station, Wilmington, Del. 19898.

${ }^{1}$ R. S. Pindak, C. C. Huang, and J. T. Ho, Phys. Rev. Lett. 32,43 (1974).

${ }^{2}$ L. Leger, Phys. Lett. 44A, 535 (1973).

${ }^{3}$ H. Birecki, R. Shaetzing, F. Rondelez, and J. D. Litster, Phys. Rev. Lett. 36, 1376 (1976).

${ }^{4}$ M. Delaye, R. Ribotta, and G. Durand, Phys. Rev. Lett. 31, 443 (1973).

${ }^{5}$ P. E. Cladis, Phys. Rev. Lett. 31, 1200 (1973).

${ }^{6}$ L. Cheung, R. B. Meyer, and H. Gruler, Phys. Rev. Lett. 31, 349 (1973).

${ }^{7}$ C. C. Huang, R. S. Pindak, P. J. Flanders, and J. T. Ho., Phys. Rev. Lett. 33, 400 (1974).

${ }^{8}$ K. C. Chu and W. L. McMillan, Phys. Rev. A 11,1059 (1975).

${ }^{9}$ W. L. Mc Millan, Phys. Rev. A $\underline{9}, 1720$ (1974) and Ref. 8.

${ }^{10}$ P. G. de Gennes, Solid State Commun. 10, 753 (1972); F. Jähnig and F. Brochard, J. Phys. 35, 301 (1974).

${ }^{11}$ P. E. Cladis, Phys. Lett. $\underline{4 \mathrm{~A}}, 179$ (1974).
${ }^{12}$ D. Langevin, J. Phys. 36, 745 (1975).

${ }^{13}$ D. Langevin and M. A. Bouchiat, J. Phys. $\underline{33}, 101$ (1972).

${ }^{14}$ M. J. Stephen and J. P. Straley, Rev. Mod. Phys. $\underline{46}$, 661 (1974).

${ }^{15} \mathrm{C}$. H. Sohl and K. Miyano, Phys. Rev. A 20, 616 (1979).

${ }^{16}$ P. G. de Gennes, The Physics of Liquid Crystals (Clarendon, Oxford, 1974).

${ }^{17}$ Ref. 14, Eq. (11.15).

${ }^{18}$ F. M. Leslie, Q. J. Mech. Appl. Math. 19, 357 (1966).

${ }^{19}$ Ref. 14, Eqs. (11.31) and (11.33).

${ }^{20}$ Ref. 14, Eq. (11.30).

${ }^{21}$ M. A. Jenkins and J. F. Traub, Numer. Math. 14, 252 (1970).

${ }^{22}$ R. H. Katyl and U. Ingard, Phys. Rev. Lett. 19, 64 (1967).

${ }^{23}$ A. E. White, P. E. Cladis, and S. Torza, Mol. Cryst. Liq. Cryst. $\underline{43}, 13$ (1977).

${ }^{24}$ S. Krishnaswamy and R. Shashidhar, Mol. Cryst. Liq. Cryst. 38, 353 (1977).

${ }^{25} \mathrm{C}$. H. Sohl, K. Miyano, and J. B. Ketterson, Rev. Sci. Instrum. $\underline{49}, 1464$ (1978). 
${ }^{26} \mathrm{~J}$. Adin Mann, Jr. and R. S. Hansen, J. Colloid. Sci. $\underline{18}$, 759 (1963).

${ }^{27}$ M. G. J. Gannon and T. E. Faber, Philos. Mag. A37, 117 (1978).

${ }^{28} \mathrm{D}$. Langevin and M. A. Bouchiat, J. Phys. $\underline{33}, \mathrm{Cl}-77$ (1972).

${ }^{29} \mathrm{~S}$. Bhattacharya (private communication).

${ }^{30}$ See, for instance, J. Als-Nielsen et al., Phys. Rev.
Lett. 39, 352 (1977); H. Birecki and J. D. Litster, Mol. Cryst. Liq. Cryst. 42,33 (1977) and references therein. ${ }^{31}$ S. Torza and P. E. Cladis, Phys. Rev. Lett. 32, 1406 (1974).

${ }^{32}$ C. H. Sohl, K. Miyano, J. B. Ketterson, and G. Wong, Bull. Am. Phys. Soc. 24, 251 (1979).

${ }^{33} \mathrm{D}$. Langevin, Phys. Lett. 56A, 61 (1976). 